\title{
Keplerian masses for the most massive stars: the very luminous, hydrogen-rich Wolf-Rayet stars
}

\author{
Anthony F. J. Moffat, Olivier Schnurr, André-Nicolas Chené, \\ Jules Casoli, Nicole St-Louis and Alfredo Villar-Sbaffi \\ Département de physique, Université de Montréal, \\ C.P. 6128, Succ. C-V, Montréal, QC, H3C 3J7, Canada \\ email: moffat, schnurr, chene, casoli, stlouis, alfredo@astro.umontreal.ca
}

\begin{abstract}
We propose that the most massive stars in the local Universe start out on the main sequence being extremely luminous and already exhibiting wind-like emission lines. Such stars can probably be identified as hydrogen-rich WN5-7 stars. Masses are now being determined for these stars using the least model-dependent technique of binary orbits.
\end{abstract}

Keywords. Binaries: spectroscopic, stars: early-type, fundamental parameters, stars: Wolf-Rayet

Locating the most massive stars and measuring their masses is key to understanding the formation and nature of massive stars in general. This is best done by using the least model-dependent technique, i.e. Kepler's laws (combined with techniques such as photospheric or wind eclipses, polarimetry, or wind-wind collision effects, to extract the orbital inclination) for binary systems that contain very luminous stars on or near the main sequence, where interaction effects are not (yet) important. In the local Universe, it appears that the potentially most massive, main-sequence stars are the most luminous hydrogen-rich WR stars of type WN5-7ha, which are even more luminous than, and probably at least as hot as, the recently recognized hottest main-sequence stars of type O2. WN5-7ha stars exhibit strong, broad WR-like emission lines because of the relatively strong winds they drive as a result of their high luminosity, not because of their compactness and high ratio of $\mathrm{L} / \mathrm{M}$ as classical He-burning, WR stars. It is probably no coincidence that the currently most massive stars measured this way are in WR 20a, an eclipsing binary system containing two identical WN6ha stars of 83 and $82 \mathrm{M}_{\odot}$ (Rauw et al. 2004; Bonanos et al. 2004; Rauw et al. 2005). Other WN5-7ha binary stars (e.g., NGC 3603/A1, 30 Dor/R145), some even more luminous than WR 20a and with masses over $100 \mathrm{M}_{\odot}$, are being measured currently by different groups, including ourselves.

\section{Acknowledgements}

We are grateful to NSERC (Canada) and FQRNT (Québec) for financial support.

\section{References}

Bonanos, A. Z., Stanek, K. Z., Udalski, A., Wyrzykowski, L., Żebruń, K., Kubiak, M., Szymański, M.K., Szewczyk, O., Pietrzyński, G., \& Soszyński, I. 2004, ApJ (Letters), 611, L33

Rauw, G., De Becker, M., Nazé, Y., Crowther, P. A., Gosset, E., Sana, H., van der Hucht, K. A., Vreux, J.-M., \& Williams, P. M. 2004, $A \mathscr{E} A$ (Letters), 420, L9

Rauw, G., Crowther, P. A., De Becker, M., Gosset, E.; Nazé, Y., Sana, H., van der Hucht, K. A., Vreux, J.-M., \& Williams, P. M. 2005, A\& $A$, 432, 985 